\title{
Evidence of Lower Macular Pigment Optical Density in Chronic Open Angle Glaucoma
}

\author{
Estera Igras \\ Mater Misericordiae Hospital, Dublin \\ James Loughman \\ Technological University Dublin, james.loughman@tudublin.ie \\ Matthew Ratzlaff \\ Mater Misericordiae Hospital, Dublin
}

See next page for additional authors

Follow this and additional works at: https://arrow.tudublin.ie/otpomart

Part of the Optometry Commons

\section{Recommended Citation}

Igras, E.,. et al: Evidence of Lower Macular Pigment Optical Density in Chronic Open Angle Glaucoma British Journal of Ophthalmology 2013, vol.97, pp994-998. doi:10.1136/bjophthalmol-2013-303153

This Article is brought to you for free and open access by ARROW@TU Dublin. It has been accepted for inclusion in Articles by an authorized administrator of ARROW@TU Dublin. For more information, please contact arrow.admin@tudublin.ie, aisling.coyne@tudublin.ie, gerard.connolly@tudublin.ie.

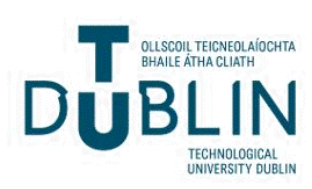




\section{Authors}

Estera Igras, James Loughman, Matthew Ratzlaff, Ronal O'Caoimh, and Colm O'Brien

This article is available at ARROW@TU Dublin: https://arrow.tudublin.ie/otpomart/32 


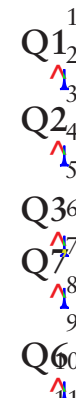

认̂̀

$\mathrm{Q}^{4^{2}}$

13

14

15

16

17

18

19

20

$\mathrm{Q} \mathrm{g}^{1}$

B2

23

24

25

26

27

28

29

30

31

Q92

33

34

35

36

37

\title{
Evidence of lower macular pigment optical density in chronic open angle glaucoma
}

\author{
Estera Igras, ${ }^{1}$ James Loughman, ${ }^{2,3}$ Matthew Ratzlaff, ${ }^{1}$ Rónán O'Caoimh, ${ }^{4}$ \\ Colm O'Brien ${ }^{1,5}$
}

${ }^{1}$ Department of

Ophthalmology, Mater Misericordiae University Hospital, Dublin, UK ${ }^{2}$ Optometry Department, School of Physics, College of Sciences and Health, Dublin Institute of Technology, Dublin, UK

${ }^{3}$ Faculty of Health Sciences, African Vision Research Institute, University of KwaZulu Nalal, Durban, South Africa

${ }^{4}$ Connolly Hospital, Dublin, UK ${ }^{5}$ Department of

Ophthalmology, School of

Medicine and Medical Science, University College Dublin, Dublin, UK

\section{Correspondence to} Dr James Loughman, Optometry Department, Schoo of Physics, College of Sciences and Health, Dublin Institute of Technology, Dublin 8, Ireland; james.loughman@dit.ie

Received 24 January 2013 Revised 25 March 2013 Accepted 21 April 2013

\section{To cite: Igras $\mathrm{E}_{\text {, }}$} Loughman J, Ratzlaff M, et al. Br J Ophthalmol Published Online First: [please include Day Month Year] doi:10.1136

bjophthalmol-2013-303153

\section{ABSTRACT \\ Background/aims Macular pigment (MP) plays an} important role in visual function and in the protection of the retina from oxidative damage. It is not known whether glaucoma, a progressive neurodegenerative disease of the optic nerve, is associated with alterations in MP. This study was designed to investigate the relationship, if any, between the optical density of MP optical density (MPOD) and glaucoma.

Methods 40 subjects (23 males, 17 females) with open angle glaucoma (mean age $69 \pm 11$ ), and 54 normal controls (23 males, 31 females) without ocular disease (mean age $66 \pm 11$ ), visual acuity (VA) $>6 / 18$, were recruited, and underwent a comprehensive eye examination including biomicroscopy, fundoscopy, Goldmann tonometry and visual field assessment, using the 24-2 SITA-fast algorithm on the Humphrey visual field analyser (II-i Series). MPOD, at $0.5^{\circ}$ of retinal eccentricity was determined, for all subjects, using heterochromatic flicker photometry.

Results Median (IQR) MPOD for subjects with glaucoma was $0.23(0.42)$ compared to $0.36(0.44)$ for controls. The difference in MPOD between the glaucoma cases and controls was statistically significant ( $\mathrm{z}=$ $-2.158, p=0.031)$. There was no significant correlation ( $p>0.05$ ) between MPOD and disease severity.

Conclusions These findings suggest that MPOD is lower in patients with glaucoma. Further investigation is needed to determine the significance of MP in glaucoma, its relationship to glare symptoms in glaucoma and to assess what role therapeutic strategies aimed at increasing MP levels could have in the management of glaucoma.

\section{INTRODUCTION}

Glaucoma is a progressive neurodegenerative disease of the optic nerve characterised by retinal ganglion cell death. ${ }^{1}$ This common ocular disease is associated with significant visual impairment and affects approximately 60 million people worldwide. $^{2}$ Glaucoma reduces quality of life proportional to the severity of visual field loss. ${ }^{3}$ Visual symptoms are frequent. The most common visual disabilities reported among glaucoma patients, using a visual disability questionnaire, are poor tolerance of glare, present in up to $70 \%$ of subjects, and difficulties with adaptation to different lighting levels in $54 \%$ of subjects. ${ }^{4}$

Macular pigment (MP) plays an important role in visual function, ${ }^{5-7}$ and in the protection of the retina from oxidative damage. ${ }^{8}$ MP comprises the three carotenoids: lutein, zeaxanthin and mesozeaxanthin, and is concentrated in photoreceptor axons at the macula. ${ }^{9}$ It has several proposed and established functions. The three main hypotheses around the function of MP relate to (a) antioxidant protection of the retina (the protective hypothesis), (b) visual performance (the acuity, visibility and glare hypotheses) and (c) neural function (the neural efficiency hypothesis). In its protective role, MP possesses optical and antioxidant properties, functioning actively and passively, to attenuate short wavelength light penetration and limit retinal oxidative damage. ${ }^{10}$ The evidence in support of the varied visual performance hypotheses suggests an important role for MP in central visual function. ${ }^{7}$ It is, perhaps, important to note that the effects of MP on visual performance are reliant on the combined influence of all three macular carotenoids, lutein, zeaxanthin and meso-zeaxanthin, which appear to act synergistically to provide a more pronounced and beneficial effect on vision. ${ }^{7}$ More recently, it has also been suggested that MP also plays a neurophysiological role, affecting temporal vision and the neural processing of visual information, and relating to dynamic markers of visual health and performance. ${ }^{11}$ Lutein is also found in the frontal and occipital cortices of the brain, correlating with cerebral carotenoid concentration, and has, therefore, been linked to cognitive performance. $^{12}$

The evidence in relation to the potential role of $\mathrm{MP}$ in glare reduction is particularly relevant given the prevalence of glare disability associated with glaucoma. Glare can be categorised as (a) the discomfort caused when the overall illumination is too bright for the adaptation level of the eye (discomfort glare), and (b) the contrast reducing effect of stray light on image quality, which causes a reduction in the visibility of a target within a visual scene (disability glare). Often thought to be non-specific, glare in glaucoma is associated with the severity of visual field defects and binocular visual field loss but is not directly linked to visual acuity (VA). ${ }^{12}$ The aetiology of glare symptoms, experienced by glaucoma patients, is poorly understood but may be directly related to pathological changes in neuronal mechanisms of the retina responsible for adaption processes. ${ }^{13} 14$

Apart from glaucoma, glare is reported in other retinal disorders including age-related macular degeneration (AMD), ${ }^{6} 15$ and hereditary retinal degenerations such as retinitis pigmentosa, ${ }^{5}$ both associated with lower MP. ${ }^{5}{ }^{16}$ Recent evidence suggests that, for healthy subjects, glare may occur as a direct consequence of MP loss. Low MP levels are associated with slower photostress recovery, increased disability glare and higher levels of visual discomfort. $^{17}$ Furthermore, oral carotenoid 
supplementation significantly increases MP and improves visual performance under glare conditions. ${ }^{7}$ This improvement in normal subjects has also been demonstrated in pathological disorders associated with glare, including AMD. ${ }^{15}$

To date, there is no published evidence linking glaucoma and MP. Given the proposed neurophysiological role of MP and the link between MP and glare intolerance in other retinal disorders, this study was designed to investigate if there is any relationship between MP optical density (MPOD) and glaucoma.

\section{MATERIALS AND METHODS Subjects}

Forty subjects undergoing treatment for established glaucoma were recruited from a glaucoma clinic at the Mater Hospital, Dublin, Ireland. Glaucoma was defined as the presence of optic nerve damage (cup to disc ratio $>0.6$ or rim notching) and reproducible glaucomatous visual field defects. Exclusion criteria included VA $<6 / 18$ and history or evidence of other retinal pathology, and use of MP supplements within the past year. An additional 54 normal controls were also recruited to participate in this case-control study, and were excluded if ocular examination revealed any signs of ocular pathology or VA $<6 / 18$. Typically the control group was composed of spouses and caregivers of the glaucoma control participants. Ethical approval was obtained from the Research Ethics Committees at Dublin Institute of Technology and the Mater Hospital, Dublin. Subjects provided informed consent prior to inclusion in the study. The study adhered to the tenets of the Declaration of Helsinki.

Demographic information, including age, gender, history of smoking (current smoker, ex-smoker and never smoked), presence of glare (subjective glare: do you suffer from glare, yes or no?) and years diagnosed with glaucoma, was collected in relation to each subject. One eye was selected for measurement of MPOD. For glaucoma subjects and controls, the eye chosen was randomly selected, except in cases where the second eye did not meet the inclusion criteria. Subjects underwent a comprehensive eye examination including VA, biomicroscopy, fundoscopy, intraocular pressure assessment and visual field analysis. Current (corrected where necessary) VA was determined using a Snellen chart. Tonometry was measured using a Goldmann tonometer. Visual fields were determined using the 24-2 SITA-fast algorithm on the Humphrey Visual Field Analyzer, II-i Series (Carl Zeiss Meditec, Jena, Germany). The severity of glaucomatous visual field loss was classified using the mean deviation (MD) value generated by the device software, into mild $(\mathrm{MD}>$ $-6 \mathrm{~dB})$, moderate (between -6 and $-12 \mathrm{~dB}$ ) and severe $(<$ $-12 \mathrm{~dB}$ ) glaucoma.

\section{Measurement of MPOD}

MPOD, at $0.5^{\circ}$ of retinal eccentricity, was determined using Heterochromatic Flicker Photometry (HFP), measured using the Macular Metrics Clinical Densitometer (Macular Metrics, Rehoboth, Massachusetts, USA). The technique involves the square-wave and counterphase presentation of two $1^{\circ}$ lights on a $10^{\circ}$ blue background field, perceived as a flickering disk by the viewer. One light is a monochromatic LED, at $458 \mathrm{~nm}$ (the peak absorbance wavelength for MP) light, whereas the other is a monochromatic LED at $550 \mathrm{~nm}$ (a wavelength that MP does not absorb). HFP was conducted by a trained examiner. All test subjects were naïve to testing. The examiner explained the procedure in detail, and provided a demonstration of the technique to each subject prior to the commencement of test. A customised HFP procedure was adopted, whereby flicker frequency was optimised for each participant prior to measurement of MPOD. The subject's task was to adjust, using a press button, the radiance of the $458 \mathrm{~nm}$ component of the test stimulus in such a way as to eliminate the flicker from the disk. This occurs when the 458 and $550 \mathrm{~nm}$ lights have the same effective luminance for the subject. The relative energy for flicker elimination at this retinal locus is then compared with the relative energy needed for flicker elimination at a location in the retina that contains no optically detectable MP (ie, $7^{\circ}$ retinal eccentricity). The log ratio of the difference between the central $0.5^{\circ}$ measure and reference $7^{\circ}$ measure yields a measure of MPOD at the test locus. As the HFP technique is not dependent on or affected by regional variations in sensitivity at the central and peripheral retinal loci (either normal or disease induced variations), the HFP technique remains appropriate for MPOD measurement among glaucoma subjects.

\section{Statistical analysis}

All data were entered into SPSS V.18.0. The data were tested for normality using the Shapiro-Wilk test. Most of the data were normally distributed and were analysed using student $t$ tests and Pearson correlation coefficient. Non-normally distributed data were analysed using a Mann-Whitney $U$ test. Median values were used when data were non-normally distributed. A general linear model was used to determine the effect of ocular health status (glaucoma or control), age, gender, smoking, glaucoma severity and years with glaucoma on the dependent variable, MP.

Assuming a 5\% level of significance, and two-tailed tests, with 40 glaucoma cases and 54 normal controls, the study power was 0.81 for detecting a difference of half of one SD between groups.

\section{RESULTS}

\section{Demographics}

A total of 40 subjects, 23 males (57.5\%) and 17 females $(42.5 \%)$ with glaucoma were included in this study. A further 54 controls (female: $n=31$; male: $n=23$ ) were also recruited. VA ranged between 6/4 and 6/9 for subjects and controls and was not statistically significantly different between groups. The median length of time since diagnosis of glaucoma was 6 years, IQR 13 years, with a range of $1-30$ years. The most common glaucoma diagnosis was primary open-angle glaucoma, $n=36$. There was one case each of pseudoexfoliative syndrome, pigmentary dispersion glaucoma, Fuchs heterochromic iridocyclitis and normal-tension glaucoma. Of note, 55\% $(n=22)$ of subjects with glaucoma stated that they suffered from glare. None of the controls reported glare. The left eye was analysed for $40 \%$ $(n=16)$, right eye for $60 \%(n=24)$ of glaucoma subjects. About $54 \%(n=29)$ of controls had their left eye analysed.

There was no statistically significant difference in gender between cases of glaucoma and controls, $t=-1.4, p=0.16$. The mean age of subjects with glaucoma was $69( \pm 11)$ years, compared with $66( \pm 11)$ years for controls. There was no statistically significant difference in the age profiles of cases and controls, $t=1.35, p=0.18$. There was no significant difference in smoking habits between glaucoma cases and controls, $\mathrm{t}=0.69, \mathrm{p}=0.49$.

\section{MPOD and glaucoma}

The median (IQR) MPOD for subjects with glaucoma was 0.23 (0.42), compared to $0.36(0.44)$ for controls. The difference in MPOD between the glaucoma cases and controls was statistically significant $(\mathrm{z}=-2.16, \mathrm{p}=0.03)$. A generalised linear model 
analysis confirmed a significant effect of ocular health status (glaucoma vs control) on MPOD $(p=0.04)$, but no effect of age, gender, smoking, years with glaucoma, or glaucoma severity on MPOD ( $p=0.2-0.97)$. The distribution of MPOD values, for glaucoma cases and normal controls, is presented in figure 1.

A Pearson correlation coefficient showed a non-significant, weak association between MPOD and MD $(r=-0.1, p=0.64)$, as demonstrated in figure 2. There was also a weak, nonsignificant correlation between MPOD and years since diagnosis of glaucoma $(r=0.16, p=0.7)$.

Test-retest repeatability of the MPOD measurement, was determined by measuring MPOD on two separate occasions, 1 week apart, for a sample of cases $(n=11)$ and controls $(n=9)$, chosen by simple randomisation. The mean $( \pm S D)$ MPOD values for the initial versus the repeat test are presented in table 1 , and demonstrate little change between tests.

There was a strong and statistically significant $(p<0.01$ for all) test-retest Pearson correlation for the combined group $(\mathrm{r}=0.84)$, normal controls $(\mathrm{r}=0.82)$ and glaucoma cases $(r=0.86)$. Paired $t$ test analysis revealed no statistically significant differences between repeat measures for the combined group $(p=0.59)$, normal controls $(p=0.42)$ and glaucoma cases $(p=0.99)$. Bland Altman analysis, including limits of agreement, revealed acceptable and equivalent levels of agreement between repeat measures for both groups (coefficient of repeatability, calculated as 1.96 times the SD of the difference between repeat measures, was 0.25 optical density units for the glaucoma group, which was marginally better than that observed for the control group, at 0.27 optical density units.)

\section{DISCUSSION}

The median MPOD of the normal control group obtained here $(0.36)$ is in general agreement with normative data from previous studies, where MPOD typically averages around 0.35 . Our findings suggest, however, that despite considerable overlap between groups, central MPOD is significantly lower in patients with glaucoma when compared to those age-matched and lifestyle-matched controls. The magnitude of the difference in MPOD between glaucoma cases and controls (MPOD 34\%

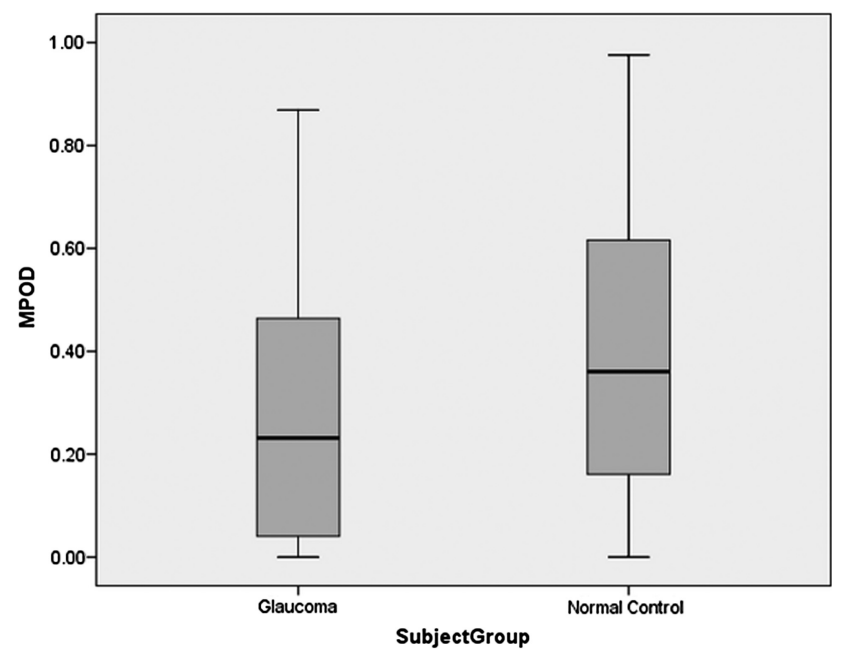

Figure 1 Box and whisker plot showing the distribution of macular pigment optical density (MPOD) for normal controls and for subjects with glaucoma. lower in glaucoma cases on average) is comparable to that observed for other conditions, where oxidative stress is a likely contributory factor, such as AMD, where magnitude differences up to $53 \%$ have been reported. ${ }^{18}$ The repeatability of the testretest MPOD values is also similar to that observed elsewhere among normal observers using the HFP technique, ${ }^{19}$ and provides further assurance as to the validity of the MPOD differences observed.

The relationship, if any, between glaucoma and MP is likely complex. The absence of any association between number of years since diagnosis or to extent of visual field loss, as categorised according to the MD index, would suggest that lower MPOD is not simply related to glaucoma severity. It is likely, however, that the SITA-Fast 24-2 programme used here (and routinely in glaucoma investigation), and the MD index reported, is not the best indicators of any functional relationship that may exist between glaucoma and MP. Detailed analysis of the central $10^{\circ}$, with particular emphasis at and close to fixation, where MPOD typically peaks would seem more appropriate for future investigations.

The absence of any gender, age or smoking habit differences between the two study groups provides a substantial degree of experimental control for those known determinants of MPOD. It should be noted, however, that dietary carotenoid intake or serum carotenoid levels were not quantified in the current study. It is certainly possible that differences in dietary intake could explain the MPOD differences observed herein. We suggest, however, that the specific recruitment into the normal control group of spouses and carers of glaucoma case participants likely limits the possibility of substantial differences in dietary intake between groups.

Although there is no published evidence linking changes in MP to glaucoma, there is growing evidence to suggest that glaucoma could create a suitable environment for MP loss. We propose two possible causative mechanisms whereby MPOD may be lower in cases of glaucoma. The first mechanism relates to glaucoma-induced changes in both the retinal

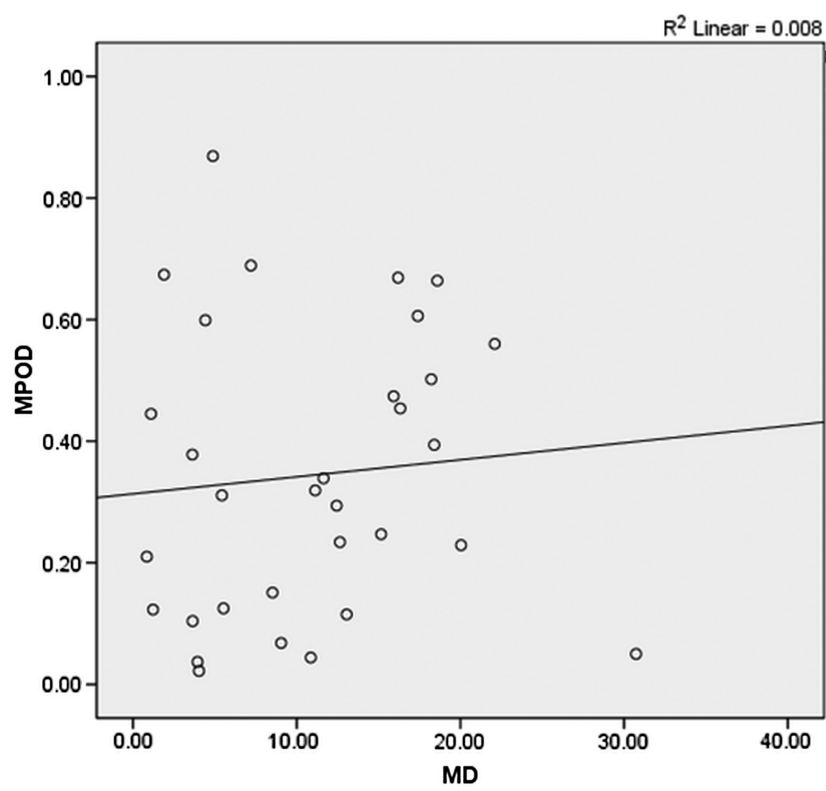

Figure 2 Scatter plot demonstrating the lack of association between macular pigment optical density (MPOD) and the severity of glaucoma, as measured using the mean deviation (MD) of visual fields for glaucoma subjects. 
Table 1 Mean $( \pm S D)$ MPOD measures for initial and repeat tests

\begin{tabular}{lll}
\hline & MPOD initial visit & MPOD repeat visit \\
\hline Normal controls $(n=9)$ & $0.39( \pm 0.24)$ & $0.36( \pm 0.18)$ \\
Glaucoma cases $(n=11)$ & $0.26( \pm 0.25)$ & $0.26( \pm 0.26)$ \\
Combined Group $(n=20)$ & $0.32( \pm 0.25)$ & $0.30( \pm 0.23)$ \\
\hline MPOD, macular pigment optical density.
\end{tabular}

microcirculation and macrocirculation sufficient to disrupt MP processing. MP is known to be entirely of dietary origin and associated with dietary intake. Dietary supplementation with macular carotenoids results in significant increases in serum carotenoid and MPOD levels, ${ }^{20}$ suggesting that the retinal microcirculation is important in MP transport to the eye. Glaucoma is associated with a reduction in ocular blood flow greater than expected for age which is, in part, related to increased resistance to blood flow. ${ }^{21}$ Atherosclerosis, vasospasm and endothelial dysfunction are all postulated to contribute to vascular regulatory dysfunction in glaucoma. These changes may influence deposition and distribution of MP. Supporting this, several fundal pigments are affected by glaucoma, including oxy-haemoglobin, melanin, xanthophyll and rhodopsin. Microcirculatory damage has been implicated in their loss. ${ }^{23}$ Macrocirculatory changes, affecting the ophthalmic, central retinal and posterior ciliary arteries, are also implicated as a causative mechanism in glaucoma. ${ }^{24}$ Furthermore, a wide variety of vascular risk factors contribute to reduced quality of blood supply, resulting in ganglion cell death at the optic nerve. $^{25}$

The second likely causative mechanism relates the increased oxidative stress associated with glaucoma to MP loss. There is strong evidence that oxidative processes damage the retina, which is susceptible to oxidative stress because of its high metabolic activity and consequential oxygen consumption, high proportion of polyunsaturated fatty acids, and constant exposure to visible light. ${ }^{9}$ Retinal pigment epithelial cells are particularly susceptible to mitochondrial DNA damage under oxidative conditions. $^{26}$ Over recent years, there is growing evidence that glaucoma causes significant free radical and reactive oxygen species (ROS) production. ${ }^{27}$ In particular, toll-like receptor signalling is upregulated in glaucoma, leading to increased cytokine production, creating a pro-inflammatory state, which may contribute to retinal ganglion cell death. ${ }^{28}$ ROS, concentrated at the trabecular membrane, are important in reducing antioxidants. ${ }^{29}$ Given the susceptibility of MP to oxidative damage, the oxidative environment created by glaucoma may also cause MP loss.

Although glaucoma is traditionally associated with peripheral visual field loss, recent technological advances have facilitated the observation that glaucoma also causes macular ganglion cell complex (GCC) loss, close to the fovea, even in its early stages. ${ }^{30}$ It is plausible that the reduced macular thickness and associated GCC loss observed in early glaucoma may be associated with simultaneous loss of MP. Such structural changes at the macula in glaucoma have also been associated with visual functional losses. Despite normal VA and visual fields, colour vision defects, ${ }^{31}$ and electrophysiological losses have also been reported, ${ }^{31}$ further implicating glaucoma in central visual and MP loss.

The prevalence of glare in the glaucoma group (55\%), compared to the normal control group $(0 \%)$ is similar to that observed in previous studies, and is also of interest. ${ }^{5}$ Given the growing connection between MP, disability glare, and visual discomfort, ${ }^{7}{ }^{17}$ it is interesting to consider the possible connection between lower MP and glare in glaucoma. Future research should include assessment of glare using objective measures and questionnaires validated in patients with glaucoma such as the Glaucoma Symptom scale. Although the benefits of carotenoid supplementation in diseases such as AMD have not definitively been proven, there is good evidence that supplementation enhances MP levels. ${ }^{20}$ The potential for therapeutic intervention, including for glaucoma, is also interesting, particularly given that carotenoid supplementation in healthy subjects reduces glare intolerance. ${ }^{7}$

In summary, this pilot study represents a preliminary investigation into MPOD in glaucoma. The results, which demonstrate lower MPOD in subjects with glaucoma, support the proposed hypothesis that circulatory changes or oxidative stress in glaucoma could affect MP. Further research is required to demonstrate if MPOD is truly lower in glaucoma (with stricter control for dietary variations in MP consumption) and if so, what are the possible aetiologies (including any effect of treatment type such as surgery or drug class), and whether the extent of reduction correlates reliably to loss of visual performance and especially glare.

Contributors $J \mathrm{~L}$ and $\mathrm{CO}^{\prime} \mathrm{B}$ contributed substantially to the conception and design of the study, to revisions of the article content, and to the final approval of the version to be published. El, MR and $\mathrm{DRO}^{\prime} \mathrm{C}$ contributed substantially to the acquisition of data, to the initial article drafting and final approval of the version to be published.

Competing interests None.

Ethics approval Dublin Institute of Technology Research Ethics Committee.

Provenance and peer review Not commissioned; externally peer reviewed.

\section{REFERENCES}

1 Gupta N, Yücel YH. Glaucoma as a neurodegenerative disease. Curr Opin Ophthalmol 2007;18:110-14.

2 Quigley HA, Broman AT. The number of people with glaucoma worldwide in 2010 and 2020. Br J Ophthalmol 2006;90:262-7.

3 McKean-Cowdin R, Wang Y, Wu J, et al. Impact of visual field loss on health-related quality of life in glaucoma: the Los Angeles Latino Eye Study. Ophthalmology 2008;115:941-8.

4 Nelson P, Aspinall P, O'Brien C. Patients' perception of visual impairment in glaucoma: a pilot study. Br J Ophthalmol 1999;83:546-52.

5 Loughman J, Davison P, Akkalli M, et al. Visual performance and macular pigment. J Optom 2010;3:73-89.

6 Loughman J, Akkali MC, Beatty $S$, et al. The relationship between macular pigment and visual performance. Vis Res 2010;50:1249-56.

7 Loughman J, Nolan JM, Howard A, et al. . The impact of macular pigment augmentation on visual performance using different carotenoid formulations. Invest Ophthalmol Vis Sci 2012;53:7871-80.

8 Li S, Fu Z, Ma H, et al. Effect of lutein on retinal neurons and oxidative stress in a model of acute retinal ischemia/reperfusion. Invest Ophthalmol Vis Sci 2009;50:836-43.

9 Snodderly DM, Auran JD, Delori FC. The macular pigment. II. Spatial distribution in primate retinas. Invest Ophthalmol Vis Sci 1984;25:674-85.

10 Beatty S, Koh H, Phil M, et al. The role of oxidative stress in the pathogenesis of age-related macular degeneration. Surv Ophthalmol 2000;45:115-34.

11 Renzi LM, Hammond BR Jr. The relation between the macular carotenoids, lutein and zeaxanthin, and temporal vision. Ophthalmic Physiol Opt 2010;30:351-7.

12 Vishwanathan R, Neuringer M, Snodderly DM, et al. Macular lutein and zeaxanthin are related to brain lutein and zeaxanthin in primates. Nutr Neurosci 2012;9: Epub PMID 22780947.

13 Nelson P, Aspinall P, Papasouliotis 0 , et al. Quality of life in glaucoma and its relationship with visual function. J Glauc 2003:12:139-50.

14 Ochsner $\mathrm{H}$, Zrenner E. Vision and dazzle: II. The effect of increasing luminance on visual acuity of glare sensitive patients. Klin Monatsb/ Augenheilkd 1992:200:110-17.

15 Richer S, Stiles W, Laisvyde S. Double-masked, placebo controlled, randomized trial of lutein and antioxidant supplementation in the intervention of atrophic age-related macular degeneration: the veterans LAST study (Lutein Antioxidant Supplementation Trial). Optometry 2004;75:223-9. 
16 Alexander KR, Kilbride PE, Fishman GA, et al. Macular pigment and reduced foveal short-wavelength sensitivity in retinitis pigmentosa. Vis Res 1987;27:1077-83.

17 Stringham JM, Garcia PV, Smith PA, et al. Macular pigment and visual performance in glare: benefits for photostress recovery, disability glare, and visual discomfort. Invest Ophthalmol Vis Sci 2011;52:7406-15.

18 Raman R, Biswas S, Vaitheeswaran K, et al. Macular pigment optical density in wet age-related macular degeneration among Indians. Eye (Lond) 2012;26:1052-7.

19 Loughman J, Scanlon G, Nolan JM, et al. An evaluation of a novel instrument for measuring macular pigment optical density: the MPS 9000. Acta Ophthalmologica 2012:90:e90-7.

20 Connolly EE, Beatty S, Loughman J, et al. Supplementation with all three macular carotenoids: response, stability, and safety. Invest Ophthalmol Vis Sci 2011:52:9207-17.

21 Harris A, Ciulla TA, Chung HS, et al. Regulation of retinal and optic nerve blood flow. Arch Ophthalmol 1998:116:1491-5.

22 Butt Z, O'Brien C, McKillop G, et al. Color Doppler imaging in untreated high and normal pressure open angle glaucoma. Invest Ophthalmol Vis Sci 1997:38:690-6.

23 Schweitzer D, Guenther S, Scibor M, et al. Spectrometric investigations in ocular hypertension and early stages of primary open angle glaucoma and of low tension glaucoma - multisubstance analysis. Intl Ophthalmol 1992;16:251-7.

24 Flammer J, Orgül S, Costa VP, et al. The impact of ocular blood flow in glaucoma. Prog Ret Eye Res 2002;21:359-93.
25 Osborne NN, Melena J, Chidlow G, et al. A hypothesis to explain ganglion cell death caused by vascular insults at the optic nerve head: possible implication for the treatment of glaucoma. Br J Ophthalmol 2001:85:1252-9.

26 Liang FQ, Godley BF. Oxidative stress-induced mitochondrial DNA damage in human retinal pigment epithelial cells: a possible mechanism for RPE aging and age-related macular degeneration. Exp Eye Res 2003;76:397-403.

27 McElnea EM, Quill B, Docherty NG, et al. Oxidative stress, mitochondrial dysfunction and calcium overload in human lamina cribrosa cells from glaucoma donors. Mol Vis 2011;17:1182-91.

28 Almasieh $\mathrm{M}$, Wilson AM, Morquette $B$, et al. The molecular basis of retinal ganglion cell death in glaucoma. Prog Retin Eye Res 2012;31:152-81.

29 Ferreira SM, Lerner SF, Brunzini $R$, et al. Oxidative stress markers in aqueous humor of glaucoma patients. Am J Ophthalmol 2004;137:62-9.

30 Tan O, Li G, Lu AT, et al. Mapping of macular substructures with optical coherence tomography for glaucoma diagnosis. Ophthalmology 2008;115:949-56.

31 Pacheco-Cutillas M, Edgar DF, Sahraie A. Acquired colour vision defects in glaucoma-their detection and clinical significance. $\mathrm{Br} J$ Ophthalmol 1999:83:1396-402.

32 North RV, Jones AL, Drasdo N, et al. Electrophysiological Evidence of Early Functional Damage in Glaucoma and Ocular Hypertension. Invest Ophthalmol Vis Sci 2010:51:1216-22. 